\title{
Die Confessio Augustana und die Einheit der Kirche
}

\section{Einleitung}

Der evangelisch-lutherisch/römisch-katholische Dialog hat sich zwar in den vergangenen mehr als fünfzig Jahren unterschiedlichen Fragen gewidmet, war dabei aber immer auf das Ziel der sichtbaren Einheit ausgerichtet. Um dieses Ziel zu erreichen, mussten die zwischen Lutheranern und Katholiken seit dem 16. Jahrhundert umstrittenen Fragen aufgegriffen und bearbeitet werden, damit tragfähige Konsense erzielt werden. Auf deren Grundlage sollte und soll die entstandene Kirchentrennung überwunden und Wege der gegenseitigen Anerkennung beschritten werden. Am Ende dieses Weges steht nach gemeinsamer lutherischkatholischer Überzeugung die Erklärung und Verwirklichung einer lutherischkatholischen Kirchengemeinschaft, die eine Gemeinschaft in der Verkündigung des Evangeliums, der Sakramente, des Amtes und der Kirche umfasst. Wie konkret diese Gemeinschaft aussehen könnte, lässt sich heute noch nicht abschließend erfassen. Lutheraner und Katholiken wissen sich aber bereits jetzt auf dem Weg zu einer wachsenden Gemeinschaft. Insoweit wird die Einheit der Kirche als eine Gemeinschaft von Kirchen verstanden, die bezogen auf die sichtbaren Zeichen ihrer Gemeinschaft ganz unterschiedliche Formen und Gestalten von Liturgien, Theologien und Lebensformen entfaltet.

Auf diesem gemeinsamen Weg zur größeren und engeren Gemeinschaft untereinander spielte und spielt die 1530 von theologischen Reformkräften der Wittenberger Bewegung verfasste Confessio Augustana (CA) eine wesentliche und wegweisende Rolle. Für den lutherisch-katholischen Dialog hat die Frage einer gegenseitigen Anerkennung der Confessio Augustana daher eine herausragende Rolle gespielt und spielt sie immer noch. Im Umfeld des 450. Gedenkjahres 1980 konnten verschiedene lutherisch-katholische Arbeitsgruppen und Kommissionen hierzu durchaus wegweisende Dokumente vorlegen. ${ }^{1}$ Dabei stand die Betonung

1 Vgl. H. Meyer/H.-J. Mund/H. Schütte (Hg.), Katholische Anerkennung des Augsburgischen Bekenntnisses? Ein Vorstoß zur Einheit zwischen katholischer und lutherischer Kirche, Frankfurt a. M. 1977 (ÖkPer 9); E. Iserloh (Hg.), Confessio Augustana und Confutatio. Der Augsburger Reichstag 1530 und die Einheit der Kirche, Münster 1980 (RST 118); H. Meyer/H. Schütte (Hg.), Confessio Augustana - Bekenntnis des einen Glaubens. Gemeinsame Untersuchung lutherischer und katholischer Theologen, Paderborn/Frankfurt a. M. 1980; K. Lehmann/E. Schlink (Hg.),

Ә OpenAccess. (c) 2022 Wolfgang Thönissen, publiziert von De Gruyter. (cc))BY-NC-ND Dieses Werk ist lizenziert unter einer Creative Commons Namensnennung - Nicht kommerziell - Keine Bearbeitung 4.0 International Lizenz. https://doi.org/10.1515/9783110683868-030 
der katholischen Intention des lutherischen Bekenntnisses im Vordergrund historischer und systematischer Abhandlungen und ökumenischer Bewertungen. Gefragt wurde dabei vorrangig nach Orientierungen im Blick auf die Zielperspektive des lutherisch-katholischen Dialogs. Welche Einheit nimmt die CA in den Blick? Welche Formen und Gestalten der Einheit lassen sich aus der CA für die Frage einer lutherisch-katholischen Kirchengemeinschaft entnehmen? Der hier vorgelegte Beitrag sucht die historische und ökumenische Rolle und die Bedeutung der CA im Kontext des lutherisch-katholischen Dialogs zu erörtern. Ausdrücklich ist keine historisch-kritische Analyse entsprechender Passagen der CA angestrebt worden.

\section{Die Confessio Augustana in der gemeinsamen historischen Beurteilung}

1530 war „die Einheit der abendländischen Kirche zwar eminent bedroht, aber noch nicht zerbrochen“2. Die Verschärfung der Gegensätze in Glauben, Lehre und Praxis der Kirche führte jedoch in der Folgezeit zur wachsenden Entfremdung, zur Vertiefung der Unterschiede und schließlich im Laufe von Jahrzehnten zur Spaltung der abendländischen Kirche. Dieser historischen Einschätzung folgend kommt die internationale lutherisch-katholische Kommission in ihrer Stellungnahme von 1980 zu der Erkenntnis: „Es ist die erklärte Absicht des Augsburgischen Bekenntnisses, den Glauben der einen, heiligen, katholischen und apostolischen Kirche zu bezeugen“3 . Der CA geht es nicht um Gründung einer neuen Kirche, sondern um „Reinerhaltung und Erneuerung des christlichen Glaubens“ ${ }^{\text {4 }}$. Dieses Urteil beruht auf historischen Untersuchungen lutherischer und katholischer Theologen. Die CA erbringt in einem hohen Maße eine Übereinstimmung in „der Mitte des christlichen Glaubens, des Heils, das Gott durch Jesus Christus im Heiligen Geist für die Welt gewirkt hat “5 Diese Übereinstimmung lässt sich auf ein gemeinsames Verständnis der Rechtfertigungslehre des Augsburgischen Bekenntnisses zurückführen, zugleich aber muss eine Differenz bei den Themen

Evangelium - Sakramente - Amt und die Einheit der Kirche. Die ökumenische Tragweite der Confessio Augustana, Freiburg i.B./Göttingen 1982 (DiKi 2).

2 So die Stellungnahme der Gemeinsamen Römisch-katholischen/Evangelisch-lutherischen Kommission zum Augsburgischen Bekenntnis, Alle unter einem Christus, 1980, Nr. 2 (DwÜ 1, 323). 3 Alle unter einem Christus, Nr. 10 (DwÜ 1, 325).

4 Alle unter einem Christus, Nr. 10 (DwÜ 1, 325).

5 Confessio Augustana - Bekenntnis des einen Glaubens, in: Meyer/Schütte, Bekenntnis des einen Glaubens, 336. 
festgestellt werden, die sich auf das Kirchenverständnis beziehen. Hier bestanden in der Vergangenheit schwerwiegende Kontroversen.

Wenn die Augsburgische Konfession ,als zentrales lutherisches Bekenntnis zugleich die am meisten auf Versöhnung angelegte und am wenigsten polemische der lutherischen Bekenntnisschriften ist" , 6 so wird sie katholischerseits verstanden als Ausdruck der Einigungsbemühungen, soweit sie „das Profil des Luthertums gleichsam in Ruhehaltung, unentstellt durch die Züge der Erregung, des Misstrauens und des Streites“ und deshalb „die am meisten authentische Gestalt

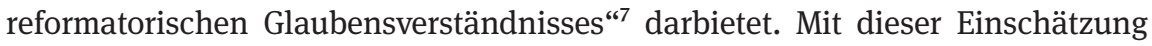
hängt auch die Beurteilung Melanchthons zusammen: Indem Melanchthon den reformatorischen Ansatz von zeitbedingten polemischen Zuspitzungen und antithetischen Fixierungen auf partikuläre spätmittelalterliche Traditionen löste, öffnete er diesen und damit auch das Hauptanliegen der Reformation, die Rechtfertigungslehre, auf die von Anfang an intendierte Universalität, Katholizität und Ökumenizität. ${ }^{8}$ Unter diesen theologiegeschichtlichen Voraussetzungen erscheint das Urteil plausibel, dass man sich nie so nahe gewesen sei wie 1530 in Augsburg. ${ }^{9}$ Der heute festgestellte Konsens im Verständnis der Rechtfertigungslehre lässt sich somit auch im historischen Rückblick festmachen. Gerade in der gemeinsamen Besinnung auf das Augsburgische Bekenntnis hat sich Katholiken und Lutheranern „ein gemeinsames Verständnis in grundlegenden Glaubenswahrheiten“"10 erschlossen. Schließt der Konsens in der Rechtfertigungslehre auch den Konsens in der Ekklesiologie mit ein?

\section{Die Bedeutung und der Rang der Rechtfertigungslehre für die Einheitsfrage}

In ihrem ursprünglichen historischen Kontext betrachtet ist die CA ,die durch das Ausschreiben des Augsburger Reichtages von 1530 herausgeforderte zusammenfassende Rechenschaft über die theologischen Einsichten der Lutherischen Reformation““. ${ }^{11}$ Sie kann als „Zusammenfassung des tatsächlichen Ertrags der Re-

6 H. Meyer/H. Schütte, Einleitung, in: Meyer/Mund/Schütte, Katholische Anerkennung, 15.

7 Meyer/Schütte, Einleitung, 15.

8 So die Einschätzung von W. Kasper, Kirchenverständnis und Kircheneinheit nach der Confessio Augustana, in: Lehmann/Schlink, Evangelium - Sakramente - Amt, $42 \mathrm{f}$.

9 So aufgenommen nach einem Urteil Luthers von W. Kasper, Kirchenverständnis, 43.

10 Alle unter einem Christus, Nr. 17 (DwÜ 1, 326).

11 W. Lohff, Die Bedeutung der Augsburgischen Konfession für die Lutherische Kirche und ihr Verhältnis zur römisch-katholischen Kirche, in: Meyer/Schütte, Bekenntnis des einen Glaubens, 1 . 
formation überhaupt ${ }^{\text {“12 }}$ gelten. Diese Einsicht macht den Weg frei zu einer vorurteilsfreien Wahrnehmung ihrer Bedeutung für den lutherisch-katholischen Dialog unserer Tage. Insofern dieses Dokument wirklich verbindliche Lehrgrundlage in den lutherischen Kirchen ist, bildet es die Voraussetzung zu einer gemeinsamen Beschäftigung: Gemeinsam besinnen sich Lutheraner und Katholiken auf den friedensstiftenden Einheitswillen der CA. ${ }^{13}$ Die Lehre der CA ist, wie der Beschluss der Lehrartikel (1. Teil der CA) vermerkt, „in heiliger Schrift klar gegrundet und darzu gemeiner christlichen, ja auch romischer Kirchen, so viel aus der Väter Schriften zu vermerken, nicht zuwider noch entgegen ist, so achten wir auch, unsere Widersacher konnen in obangezeigten Artikeln nicht uneinig mit uns sein“. ${ }^{14}$ Wenn in der Stellungnahme der Gemeinsamen Römisch-katholischen/Evangelisch-Lutherischen Kommission zum Augsburgischen Bekenntnis im Jahre 1980 festgehalten wird, dass es die Absicht des Augsburgischen Bekenntnisses ist, „den Glauben der einen, heiligen, katholischen und apostolischen Kirche zu bezeugen, dann geht es um Reinerhaltung und Erneuerung des christlichen Glaubens in Einklang mit der Alten Kirche, ,auch der römischen Kirche und in Übereinstimmung mit dem Zeugnis der Heiligen Schrift. “ ${ }^{15}$ Die inhaltlichen Aussagen, so wird dort weiter herausgehoben, entsprechen dieser Absicht in hohem Maße. ${ }^{16}$ Insoweit können sie auch als Ausdruck des gemeinsamen Glaubens verstanden werden. In Übereinstimmung mit den Artikeln 1 und 3 der CA kann das lutherisch-katholische Dokument auch das gemeinsame Bekenntnis im Glauben an den dreieinigen Gott und die Heilstat Gottes durch Jesus Christus im Heiligen Geist zum Ausdruck bringen.

Schon in diesem frühen lutherisch-katholischen Dokument zum 450-jährigen Gedenken an die Verlesung der Augsburgischen Konfession findet sich, basierend auf der schon im sog. Malta-Bericht von 1972 angezielten Übereinstimmung, ${ }^{17}$ die für den späteren Dialog so bemerkenswerte Feststellung eines Konsenses in der Lehre von der Rechtfertigung. Dabei sind zwei Aussagen von Bedeutung: 1. Die Lehre von der Rechtfertigung war für die Reformation von entscheidender Bedeutung (Nr. 14). - Diese Aussage bezieht sich eindeutig auf die Confessio Au-

12 Lohff, Bedeutung, 12.

13 So das Ergebnis der gemeinsamen Studienarbeit, die in dem von H. Meyer und H. Schütte herausgegebenen Werk, dokumentiert wird: Meyer/Schütte, Bekenntnis des einen Glaubens, 333. 14 BSLK, 83d.

15 Alle unter einem Christus (DwÜ 1, 325).

16 Mit Bezug auf das von H. Meyer und H. Schütte herausgegebene Werk, Meyer/Schütte, Bekenntnis des einen Glaubens, 333.

17 Bericht der Evangelisch-lutherisch/Römisch-katholischen Studienkommission, Das Evangelium und die Kirche, 1972, Nr. 26f. (DwÜ 1, 255). 
gustana und bringt ihre Grundintention zur Geltung (CA 4): Die Aussagen über die Rechtfertigung in Artikel 4 schließen sich an den vorhergehenden Artikel 3 über Jesus Christus an, auf Artikel 4 folgt eine Ausführung über das Wirken des Heiligen Geistes in Artikel 5. Das aber heißt: Der Artikel über die Rechtfertigung ist christologisch begründet und pneumatologisch ausgerichtet. Von seiner ganzen Stellung her ist der Artikel von der Rechtfertigung „Mitte und Klammer des Bekenntnisses“. ${ }^{18}$ CA 4 kann deswegen auch als Hauptartikel der Confessio Augustana bezeichnet werden. ${ }^{19}$

Das aber heißt nun: Durch diese Anordnung gibt Melanchthon dem Artikel von der Rechtfertigung seine das Bekenntnis tragende Bedeutung. 2. „Allein aus Gnade und im Glauben an die Heilstat Christi, nicht auf Grund unseres Verdienstes, werden wir von Gott angenommen und empfangen den Heiligen Geist, der unsere Herzen erneuert und uns befähigt und aufruft zu guten Werken.“20 Der sich in dieser Formulierung abzeichnende Konsens, eruiert aus den Artikeln 4, 6 und 20 der CA, stellt die Grundlage für ein gemeinsames Verständnis der Rechtfertigung dar. Es ist exakt diese Formel, welche nunmehr das zentrale gemeinsame Bekenntnis in der zwischen Lutherischem Weltbund und römisch-katholischer Kirche vereinbarten Gemeinsamen Erklärung zur Rechtfertigungslehre von 1999 ausmacht (Nr. 15). Das aber heißt: Die lutherisch-katholische Verständigung erfolgt auf der Grundlage eines die gesamte Lehre und Praxis der Kirchen auf Christus hin orientierenden, unverzichtbaren Kriteriums: Die Botschaft von der Rechtfertigung verweist auf die Mitte des neutestamentlichen Zeugnisses von Gottes Heilshandeln in Christus (Nr. 17). Darin spricht sich die Einsicht aus, dass ein gemeinsames Verständnis der Rechtfertigung ein gemeinsames Verständnis der Mitte des christlichen Glaubens einschließt. ${ }^{21}$ Die Botschaft von der Rechtfertigung meint nichts anderes als das Evangelium. Somit erfasst die gemeinsame Verständigung zwischen Lutheranern und Katholiken die Grundintention der CA, und dieses Bekenntnis erlangt damit einen einzigartigen ökumenischen Rang. Damit kommt die ursprüngliche Absicht Melanchthons zur Geltung, die er im ersten Entwurf für einen Beschluss der Augsburger Konfession so formuliert hat:

Wir haben auch niemand mit dieser Schrift zu schmähen gedacht, sondern allein unser Bekenntnis getan, daraus männiglich erkennen mag, dass wir in Lahr und Zeremonien nicht halten zuwider Gottes Wort oder der heiligen gemeinen und catholica christlichen Kirchen.

18 G. Müller/V. Pfnür, Rechtfertigung - Glaube - Werke, in: Meyer/Schütte, Bekenntnis des einen Glaubens, 110.

19 Vgl. W. Maurer, Historischer Kommentar zur Confessio Augustana, Bd. 2, Gütersloh 1978, 68. 20 Alle unter einem Christus, Nr. 14 (DwÜ 1, 326).

21 Vgl. das Ergebnis der Studienarbeit zu Meyer/Schütte, Bekenntnis des einen Glaubens, 336. 
Denn das ist öffentlich, dass wir mit höchstem Fleiß gewehret haben, dass nicht neue unchristliche Lahr bei uns gelehret oder angenommen werden möcht. ${ }^{22}$

Greifen die Reformtheologen mit diesem Bekenntnis zur Lehre der Kirche etwa auf theologische Konzepte des späten Mittelalters zurück, um die Katholizität ihres eigenen rechtfertigungstheologischen Anliegens zu behaupten? In welchem Verhältnis stehen Rechtfertigungslehre und Ekklesiologie am Ende des Mittelalters zueinander? Gibt es überhaupt einen Zusammenhang?

\section{Rechtfertigung und Kirche im Mittelalter}

Die Ekklesiologie des ersten Jahrtausends zeichnet sich durch ein überaus reiches Bild an unterschiedlichen Kirchenbildern aus. ${ }^{23}$ Die von den Kirchenvätern aus der Heiligen Schrift entnommenen Symbole erschließen das Wesen der Kirche und ihrer Mysterien. Augustinus nimmt angesichts dieses reichen Erbes eine gewisse Konzentration vor, indem er die Kirche als den mystischen Leib Christi bezeichnet und damit für die folgenden Jahrhunderte die Anbindung der Ekklesiologie an die Sakramentenlehre und insbesondere der Eucharistie vorzeichnete. ${ }^{24}$ Wird so das reiche ekklesiologische Erbe des ersten Jahrtausends in das zweite hinein weitergereicht, so erkennt man, dass die in Sentenzen, Quaestiones und Summen zusammengefassten Erörterungen über das Kirchenbild weitgehend auf Augustinus zurückgehen, die neutestamentlichen Bilder und Begriffe aufgreifen und damit das Erbe der Patristik erhalten. Gleichzeitig aber ist festzustellen, dass in der Sicht der Kirche die Vorstellung von der Heilsanstalt einen zentralen Platz in den ekklesiologischen Aussagen über die Kirche einzunehmen beginnt. Sind es zunächst einmal die heilsgeschichtlichen Perspektiven, welche die Ekklesiologie der Frühscholastik bestimmen, so kann aber kaum übersehen werden, dass der hierarchische Aspekt der Einheit der Kirche durch die Unterordnung unter den Papst als dem Inhaber der Primatialgewalt viel stärker hervortritt und damit die Struktur der Kirche allmählich zu bestimmen beginnt.

22 BSLK, 136.

23 W. Knoch, „Communio Ecclesiae“. Ein Grundgedanke der Ekklesiologie des Vatikanum II im Spiegel frühscholastischer Theologie, in: J. Schreiner/K. Wittstadt (Hg.), Communio Sanctorum. Einheit der Christen - Einheit der Kirche. FS für Bischof Paul-Werner Scheele, Würzburg 1988, 425-438. Vgl. hierzu Y. M.-J. Congar, Die Lehre von der Kirche. Von Augustinus bis zum Abendländischen Schisma, Freiburg i.B. (HDG 3/3c), 171.

24 Knoch, Communio Ecclesiae, 427. 
Auch am Vorabend der Reformation lässt sich diese Tendenz klar feststellen. In dem 1517 erschienenen Theologischen Wörterbuch des Johannes Altenstaig, ${ }^{25}$ eine, wie Vinzenz Pfnür sich ausgedrückte, „letzte zusammenfassende vorreformatorische Präsentation der Positionen der mittelalterlichen und insbesondere der spätmittelalterlichen Theologie“, ${ }^{26}$ ist noch ein weiterer Aspekt zu berücksichtigen, der die vorreformatorischen Auseinandersetzungen stärker zu konturieren versteht. Greift man etwa auf den Artikel von der communio sanctorum ${ }^{27}$ zurück, den der Verfasser des Lexikons hauptsächlich mit Zitaten aus einem Schreiben des Johannes Eck füllt, so erkennt man das in den Mittelpunkt der ekklesiologischen Erörterungen gerückte Interesse an Fragen nach der Gnade und des Verdienstes des einzelnen Gläubigen:

Die Gemeinschaft des Glaubens [...] bezieht sich auf die Sakramente. So heißt es: „Ein Herr, eine Taufe“ <Eph 4,5> und diese ist gemeinsam allen Gläubigen, die an der einen Taufe teilhaben, mit einem einzigen unauslöslichen Zeichen bezeichnet sind und mit demselben Blut Christi erlöst sind. Und diese Gemeinschaft ist groß. Eine weitere ist die Gemeinschaft der Gnade, die darin besteht, dass die Gläubigen teilhaben an der Gnade, die von dem einen Haupt Jesus Christus kommt. Und diese ist allen Gerechten, die in der Gnade Gottes leben, gemeinsam und strömt ein von dem einen Haupt, dass Christus ist. Eine dritte ist die Gemeinschaft des Verdienstes: so wie jeder für sich im Besonderen aufgrund der überaus barmherzigen Großzügigkeit Gottes verdient, verdient der für den anderen im Allgemeinen. Diese Gemeinschaft bedeutet, dass das Band der Liebe so groß ist - gleichwie Gottes gütige Barmherzigkeit -, dass wenn ein Gerechtfertigter auf Erden ein gutes, verdienstliches Werk tut, Gott dieses als wesentlichen Lohn für den, der es tut, annimmt und allgemein für alle die in der Gnade sind. ${ }^{28}$

Kein Zweifel besteht angesichts des Befundes: Entgegen der Auffassung der Kirchenväter und frühen Scholastik wird das Kirchenverständnis der späten Scholastik nicht mehr vorrangig von der Eucharistiegemeinschaft her aufgefasst, sondern, wie Eck es unternimmt, auf die in der Taufe grundgelegte sakramentale

25 J. Altenstaig, Vocabularius Theologiae complectens vocabulorum descriptiones, Hagenau 1517; J. Altenstaig/J. Tytz, Lexicon Theologicum, Köln 1619 (Nachdruck: Hildesheim/New York 1974).

26 V. Pfnür, Zum Verständnis der communio in der spätmittelalterlichen Theologie, in: Schreiner/Wittstadt, Communio Sanctorum, 147-167, 148.

27 Pfnür, Zum Verständnis, 162.

28 Vel adhuc clarius potest dici ut Ioan. Eckius scribens ad Dominum Leonardum Abbatem Ottenburensem de communione sanctorum. [...] Communio fidei et respicit sacramenta [...] Alia est communio gratiae, quod fideles communicant gratia ab uno capite Iesu Christo [...] Tertia est communio meriti: ita ut dum quisque sibi meretur singulariter ex Dei misericordissima liberalitate: meretur alteri universaliter“ (J. Altenstaig, Lexicon Theologicum, 162/Übersetzung, die hier ausführlicher zitiert wird: Pfnür, Zum Verständnis, 151). 
Gemeinschaft des Glaubens bezogen. Aspekte der Eucharistie treten signifikant zurück. Dieser Eindruck wird durch weitere Artikel des Theologischen Wörterbuchs bestätigt. Die Kirche wird zwar weiterhin als congregatio sanctorum oder congregatio fidelium oder auch als corpus Christi mysticum bezeichnet, aber viel stärker treten äußere Aspekte im Kirchenverständnis hervor. Die „Kirche tritt als sakramentale Gemeinschaft des Leibes zwischen der Kirche als äußerem Gebäude und als Organisation auf der einen Seite und der Kirche als Vereinigung der Guten auf der anderen Seite“29 ${ }^{\text {z }}$ urück. Kirche wird vom Gedanken der Versammlung der Gläubigen her verstanden. Noch schärfer gesagt, treten Eucharistie und Ekklesiologie immer stärker auseinander, werden Aspekte der individuellen Heilsfrömmigkeit, die unter der Thematik der Rechtfertigung und der Gnade verhandelt werden, immer häufiger in der Betrachtung der Kirche hervorgehoben. ${ }^{30}$ Das Kirchenverständnis des Spätmittelalters wird von der Rechtfertigung- und der Gnadenlehre her bestimmt, nicht mehr vorrangig von der Sakramentenlehre und der Eucharistie. Insoweit hat die Theologie der Spätscholastik den Vorrang der Rechtfertigungslehre in der Bestimmung der Ekklesiologie bestätigt. Für die Beurteilung der reformatorischen Ekklesiologie ist die „Beachtung dieses spätmittelalterlichen Kirchenverständnisses [...] nicht unerheblich“. ${ }^{31}$ Die Ausrichtung auf das Heil des einzelnen Gläubigen ist dann zwar das durchgängige Muster der reformorientierten Theologen, sie erwägen diese Frage allerdings im Horizont der überlieferten ekklesiologischen Begrifflichkeiten. So bleibt denn hier zu fragen, ob die Reformatoren die überlieferte klassische Ekklesiologie mit ihrer Zentrierung auf die Eucharistie als Mitte und Quelle der communio sanctorum aufsprengen und letztlich aufgeben ${ }^{32}$

\section{Kontinuität im Kirchenbegriff?}

Der erste Satz von CA 7 bringt die Grundüberzeugung der Wittenberger Reformtheologen zum Ausdruck, ${ }^{33}$,dass alle Zeit musse ein heilige christliche Kirche sein und bleiben“. Wie schon an der Gesamtabsicht erkennbar, so ist auch in

29 Pfnür, Zum Verständnis, 159.

30 Vgl. H. de Lubac, Corpus Mysticum. Eucharistie und Kirche im Mittelalter. Eine historische Studie, Einsiedeln 1969, 316ff.

31 Pfnür, Zum Verständnis, 160.

32 Vgl. Jürgen Lutz, Unio und Communio. Zum Verhältnis von Rechtfertigungslehre und Kirchenverständnis bei Martin Luther, Paderborn 1990 (KKTS LV).

33 H. Meyer/H. Schütte, Die Auffassung von Kirche im Augsburgischen Bekenntnis, in: Meyer/ Schütte, Bekenntnis des einen Glaubens, 171. 
diesem Artikel 7 die CA zutiefst beherrscht von dem Gedanken der Kontinuität der Kirche. Der lateinische Text an dieser Stelle verdeutlicht das: Ecclesia perpetuo mansura, das heißt: die Kirche besteht ununterbrochen, beständig. Darin ist zugleich das Bekenntnis zur Katholizität der Kirche eingebunden. Denn der Artikel von der unter den Nationen verbreiteten „katholick oder gemein Kirchen“ hält fest, dass „eine christliche Kirche bis an das Ende der Welt auf Erden sein und bleiben werde“, wie es in der Apologie heißt. ${ }^{34}$ Aber mehr noch zeigen sich Kontinuität und Katholizität in der materialen Übereinstimmung im Kirchenbegriff selbst. Dieser manifestiert sich in der Definition von Kirche, die lautet: Est autem ecclesia congregatio sanctorum (CA 7). In der Apologie der Konfession weist Melanchthon ausdrücklich darauf hin, dass er nichts Neues gesagt habe, sondern hierzu lediglich auf Paulus und das Glaubensbekenntnis verweise, wenn er die Kirche als Gemeinschaft der Heiligen verstehe. Mit der Tradition der Kirche steht dieser Begriff durchaus in Übereinstimmung, wenngleich die CA zunächst nur den personalen Aspekt besonders betont, die Kirche sei Versammlung aller Gläubigen. Diese congregatio ist aber dadurch ausgezeichnet, dass in ihr das Evangelium gepredigt und dass in ihr die Sakramente gereicht werden. Das lateinische in qua macht hierbei deutlich, dass die Verkündigung des Evangeliums und die Sakramentenspendung in diese Versammlung eingestiftet sind. Also ist die Versammlung der Gläubigen keine Gemeinschaft, die durch Zusammenschluss gleichgesinnter Menschen aufgrund ihres Bekenntnisses oder ihrer Gläubigkeit konstituiert wird, sondern durch den in Wort und Sakrament gegenwärtigen Herrn geschaffen wird. Congregatio sanctorum meint auch hier immer noch die durch die Teilhabe an den sancta, nämlich Evangelium und Sakramente, bestimmte Gemeinschaft (so in Artikel 5). Damit bleibt der objektive Aspekt im altkirchlichen Begriff (Teilhabe an...) in Geltung. Der Boden der altkirchlichen Ekklesiologie wird nicht verlassen. An der Kontinuität zur altkirchlichen Ekklesiologie lässt Melanchthon also keinen Zweifel.

Auf dem Augsburger Reichstag haben die expliziten Aussagen der CA über die Kirche verhältnismäßig wenig Aufmerksamkeit gefunden. Denn nicht die Kirchenfrage, sondern die Heilsfrage stand im Vordergrund des Interesses. ${ }^{35}$ Die Confutatio, keineswegs eine antireformerische Gegenschrift der Altgläubigen, ${ }^{36}$ weist zwar die Definition der Kirche als congregatio sanctorum zurück, weil sie eine Gefahr für den Glauben darstelle, insofern die Sünder und Bösen aus der

34 BSLK, $235 \mathrm{f}$.

35 W. Kasper, Das Kirchenverständnis der Confessio Augustana, in: Iserloh, Confessio Augustana und Confutatio, 396.

36 Vgl. H. Immenkötter, Der Reichstag zu Augsburg und die Confutatio. Historische Einführung und neuhochdeutsche Übertragung, München 1979 (KLK 39), 30. 
Kirche ausgewiesen würden, lobt aber das Bekenntnis zur Tradition der Kirche ebenso wie den Unterschied zwischen der Einigkeit im Glauben und den verschiedenen partikularen Kirchenordnungen. ${ }^{37}$ In Melanchthons Apologie nimmt die Kirchenfrage neben den Artikeln über Rechtfertigung und Beichte dann einen außerordentlich breiten Raum ein. In den späteren Schriften des Corpus Doctrinae Philippicum wird sich das nochmals verstärken. Das weist darauf hin, dass der ekklesiologischen Frage im Fortgang der lutherischen Reformation eine wachsende Bedeutung zukam. ${ }^{38}$ Dem kommt die Anordnung des Artikels 7 ,Von der Kirche“ im Anschluss an Artikel 4 „Von der Rechtfertigung“ und Artikel 5 „Vom Predigtamt“, d.h. vom kirchlichen Amt, entgegen. Die CA stellt die ekklesiologische Frage unter den Primat des Evangeliums und bezieht sie auf die Verkündigung des Evangeliums. Das Evangelium macht die Kirche zur Kirche. Wenn das Evangelium inhaltlich als Botschaft von der Rechtfertigung bestimmt wird, so geht daraus klar hervor: Das Rechtfertigungsgeschehen selbst ist der Urgrund für das Werden und Bestehen der Kirche. Es ist genau dieser von der Confessio Augustana beschrittene Weg, der im lutherisch-katholischen Dialog unserer Tage bestätigt und angenommen wurde. Im Dokument Kirche und Rechtfertigung heißt es deshalb: „Alles, was über das Wesen der Kirche, über die Heilsmittel und über das der Kirche eingestiftete Amt geglaubt und gelehrt wird, muss im Heilsgeschehen selbst begründet und vom Rechtfertigungsglauben als Empfang und Aneignung des Heilsgeschehens geprägt sein. “" ${ }^{39}$ Die Kirchenfrage steht im engen Zusammenhang mit der Rechtfertigungslehre, und dies gilt auch umgekehrt. Weil die Rechtfertigungsbotschaft Ausdruck des universalen Heilswillens Gottes ist, ist sie der Maßstab für Struktur, Lehre und Praxis der Kirche. Die CA bringt damit aber nichts Anderes zum Ausdruck, als dass Jesus Christus der Grund der Kirche ist.

In Übereinstimmung mit dem altkirchlichen Symbolum ${ }^{40}$ hält Melanchthon auch die Kirche als „Gesellschaft äußerlicher Zeichen““41 fest, die auf Erden sein und bleiben wird. Die Kirche ist nach der Schrift und dem Zeugnis des Paulus Leib Christi. So tritt das Bekenntnis zur ecclesia visibilis später noch schärfer hervor. Fehlen Aussagen zur Kirche in den Loci communes von 1521, so treten sie umso stärker in der deutschen Fassung der Loci theologici von 1553 hervor. Melanchthon ist sich des Widerspruchs seiner Gegner bewusst und stellt deshalb klar, dass die

37 Immenkötter, Der Reichstag zu Augsburg, 52f.; lateinischer Text: Die Confutatio der Confessio Augustana vom 3. August 1530, bearb. v. H. Immenkötter, Münster 1979 (CC 33), 95.

38 Meyer/Schütte, Die Auffassung von Kirche, $168 \mathrm{f}$.

39 Kirche und Rechtfertigung, Nr. 2 (DwÜ 3, 320).

40 Melanchthon zitiert diesen Artikel ausdrücklich in ApolCA 7 (BSLK, 235).

41 ApolCA 7 (BSLK, 234, 29f.). 
Kirche als communio sanctorum eine sichtbare Kirche, eine sichtbare Versammlung ist. ${ }^{42}$ Er redet nicht „von der Kirchen als von einer Idea Platonica [...], da niemand wisse, wo sie zu finden sei“. ${ }^{43}$ Die Rede von der öffentlichen Predigt des Evangeliums ist hier so zu verstehen, dass sie auf den sichtbaren Charakter der Kirche verweist. In diesem Sinne ist die Kirche dann eine Versammlung der Heiligen und der Heuchler zugleich. Die Kirche, als sichtbare Versammlung, ist ein mixtum compositum, von Gott gestiftet, unter seinem Wort stehend, Versammlung der Heiligen, das sind die zur ewigen Seligkeit Auserwählten, die nur Gott kennt. Zugleich aber ist sie auch Versammlung von Heuchlern und Bösen und darin doch die von Gott geheiligte Versammlung. Damit ist die „rechte, warhafftige Kirche und Gottes volck“44 eine sichtbare Kirche mit äußerlichen Zeichen. Sichtbare Kirche umfasst als äußere Zeichen die reine Lehre des Evangeliums, den rechten Gebrauch der Sakramente und das von Gott eingesetzte ministerium. ${ }^{45}$ Von hier aus ist der Hinweis auf die Kirche als eine einzige komplexe Wirklichkeit, wie es das Zweite Vatikanische Konzil in der Kirchenkonstitution Lumen gentium entfaltet hat, nicht weit. Diese eine Kirche Christi ist heilig und doch stets der Reinigung bedürftig, weil sie „Sünder in ihrem eigenen Schoße“ umfasst. ${ }^{46}$ Mehr muss als Erweis einer bestimmten Kontinuität im Kirchenbegriff hierzu nicht ausgeführt werden. Sie spricht für sich selbst.

\section{Was ist zur Einheit der Kirche notwendig?}

Einen Schritt weiter führt die gleich im Anschluss an diese Definition von Kirche ausgeführte Unterscheidung von dem zur Einheit der Kirche Notwendigen bzw. Nichtnotwendigen: „Dann dies ist gnug zu wahrer Einigkeit der christlichen Kirchen, dass da einträchtiglich nach reinem Verstand das Evangelium gepredigt und die Sakrament dem gottlichen Wort gemäß gereicht werden.“ Der beherrschende Gedanke ist hierbei: „Was die Kirche zur Kirche macht, deckt sich mit dem, was zur Einheit der Kirche notwendig ist. “47 Das für Wesen und Struktur der Kirche Notwendige ist das Evangelium, das in Wort und Sakrament vermittelt und

42 Heubtartikel Christlicher Lere. Melanchthons deutsche Fassung seiner Loci Theologici, 1553, hg.v. R. Jenett/J. Schilling, Leipzig 2002, $383 \mathrm{f}$.

43 Examen ordinandorum, LXXV (MSA VI, 212).

44 Heubtartikel Christlicher Lere, 390.

45 Examen ordinandorum, LXXV (MSA VI, 212).

46 LG 8.

47 Meyer/Schütte, Die Auffassung von Kirche, 170. 
durch den Glauben angenommen wird. ${ }^{48}$ In dieser radikalen Konzentration auf die Frage nach der rechten Evangeliumsverkündigung in Predigt und Sakrament lässt Melanchthon das reformorientierte Anliegen bezüglich der Einheit der Kirche klar hervortreten. Als Kriterien für die Einheit der Kirche ergeben sich, zieht man CA 5 „Vom Predigtamt“ mit heran: Verkündigung des Evangeliums, Sakramentenspendung und das von Gott eingesetzte Amt. Der Sache nach ist nämlich das kirchliche Amt in CA 7 mitgesetzt, da das Evangelium immer nur als verkündigtes und Sakramente immer nur als dargereichte zu verstehen sind. Wort und Sakrament können gar nicht ohne das gottgesetzte Amt sein. So erscheint in dieser Perspektive nochmals der Wille zur Einheit durch, denn auch die katholische Tradition hat an den für Kircheneinheit notwendigen Bedingungen immer festgehalten. Wenngleich die CA das Problem der Lehr- und Leitungsautorität, letztlich den Unfehlbarkeitsanspruch des kirchlichen Amtes insgesamt nicht thematisiert hat, so bleibt doch erkennbar, dass auf dem Boden der CA und der Apologie ein prinzipieller Konsens in der Frage der Einheit der Kirche erreichbar scheint. Dieser Konsens wird durch Melanchthons Tractatus De potestate et primatu papae nicht aufgehoben. Auch hier weist Melanchthon mehrfach auf das der Kirche eingestiftete Amt, den ordo, hin. ${ }^{49}$ Die rechte Kirche zeichnet sich durch eine von Gott gegebene Gewalt aus, die den Pastoren, Presbytern und Bischöfen übergeben ist. ${ }^{50}$ Sie umfasst die ordentliche Gewalt, ministri zu wählen und zu ordinieren. ${ }^{51}$ Damit bestätigt Melanchthon, dass die Kirche ein „aus göttlicher ordnung ${ }^{\text {“52 }}$ kommendes „ministerium und Ampt haben muss“ ${ }^{53}$ Durch das Amt ist Gott selbst in der Kirche wirksam. ${ }^{54}$

Melanchthon zählt im Unterschied zu Luther die Ordination zu den Sakramenten. „Das ist keine zufällige Randaussage in der Apologie des Augsburgischen Bekenntnisses, sondern eine sowohl in akademischen Disputationen wie auch in den späteren Ausgaben seiner evangelischen Dogmatik, den Loci, immer wieder bestätigte Grundüberzeugung. Die mit dem Ordinationsgebet verbundene Auflegung der Hände hat für ihn einen im weiteren Sinn sakramentalen Rang. “55 Ordo de ministerio nennt Melanchthon in der deutschen Fassung der Apologie „das

48 Kirche und Rechtfertigung, Nr. 39 (DwÜ 3, 332).

49 BSLK, 479f.

50 Jus est donum propie datum ecclesiae (BSLK, 491).

51 BSLK, 491.

52 Heubtartikel Christlicher Lere, 379.

53 Heubtartikel Christlicher Lere, 382.

54 Confessio Saxonica 1551, 409: in quo Deus per ministerium Evangelii est efficax (MSA VI, 1219). 55 A. Sander, Luther und Melanchthon. Ökumenische Überlegungen zur Doppelspitze der Wittenberger Reformbewegung, in: KNA-ÖKI Nr. 27, 6. Juli 2010, 3. 
Sakrament des Ordens“; so besteht für ihn keine Beschwernis darin, „die Ordination ein Sakrament zu nennen““ ${ }^{56}$ Hierein bezieht er ausdrücklich das Auflegen der Hände. ${ }^{57}$ Die Kirche hat die Gewalt, „tüchtige Personen zum Bischofflichen Ampt, das ist zur Seelsorge, zu wehlen und inen das Ampt zu befehlen. Und ist gewöhnlich und löblich, das solchs geschehe also, das etliche Christliche und gelerte Seelsorger dabey seien und ire Lere anhören und zum Zeugnis inen die hende aufflegen. “58 Auflegung der Hände und Gebet nennt Melanchthon „Weihe und nicht anders“. 59

Dieses erstrangige Resultat historischer Forschung wird durch den ökumenischen Dialog bestätigt und rezipiert. Ausdrücklich im Anschluss an die CA bekräftigt der Ökumenische Arbeitskreis evangelischer und katholischer Theologen: Als Bedingung der Einheit darf nur verbindlich sein, „was um des Evangeliums willen notwendig ist“". ${ }^{60}$ In Bezug zu dieser durch die Rechtfertigungsbotschaft grundgelegten Bedingung bilden Evangeliumsverkündigung, Sakramentenspendung und - ihnen dienend zugeordnet - apostolisches Amt Zeichen für die Einheit der Kirche. Das lutherisch-katholische Gespräch auf Weltebene suchte in dem 1985 veröffentlichten Dokument „Einheit vor uns“ Klarheit über das Wesen kirchlicher Einheit zu erlangen. Ohne dass dies ausdrücklich vermerkt wurde, steht im Hintergrund der folgenden Ausführung die von der CA prononcierte Sicht der Einheit: „Die in Christus geschenkte, im dreieinigen Gott wurzelnde Einheit der Kirche verwirklicht sich in der Gemeinschaft im verkündigten Wort, in der Gemeinschaft in den Sakramenten und in der Gemeinschaft in dem von Gott eingesetzten und durch Ordination zu übertragenden

56 Si autem ordo de ministerio verbi intelligatur, non gravatim vocaverimus ordinem sacramentum (ApolCA 13,11, in: BSLK, 293). Weitere Belege sind: Veteres, ut magnitudinem et dignitatem ministerii commendarent hominibus, sapienter posuerunt ordinationem inter Sacramenta (CR 12, 490). Auch in den späteren Ausgaben seiner evangelischen Dogmatik, den Loci, findet man folgende Aussage: Cum autem vocabulum Sacramenti ceremoniis intelligitur institutis in praedicatione Christi, numerentur haec Sacramenta: Baptismus, Coena Domini, Absolutio, quae sunt externi ritus et sunt signa totius Evangelii [...] Mihi maxime placet etiam addi Ordinationem, ut vocant, id est, vocationem ad ministerium Evangelii et publicam eius vocationis approbationem; quia haec omnia mandato Evangelii praecipiuntur (CR 21, 848-853, hier 849f.).

57 ApolCA 13,18 (BSLK, 293).

58 Heubtartikel Christlicher Lere, 379.

59 Heubtartikel Christlicher Lere, 379.

60 Gemeinsame Erklärungen des Arbeitskreises: Zeichen der Einheit der Kirche im Anschluss an die Confessio Augustana: Evangelium - Sakramente - Amt, in: Lehmann/Schlink, Evangelium Sakramente - Amt, 184. 
Amt. “61 Darin bekundet sich wieder die zwischen Lutheranern und Katholiken gewonnene Grundüberzeugung, dass die Verkündigung des Evangeliums in Predigt und Sakramenten nur in der Kirche ihren Ort hat. ${ }^{62}$ Die Verwirklichung lutherisch-katholischer Kirchengemeinschaft, welche die Gemeinschaft im apostolischen Glauben, im sakramentalen Leben und als Dienstgemeinschaft umfasst, weiß sich damit auf die Grundintention der CA verwiesen; so ist sie auch aus dieser Perspektive in ihrem Einheitswillen erkannt.

\section{Die Bedeutung der Confessio Augustana für die Einheit der Kirche. Ökumenische Erkenntnisse}

Melanchthons gegenüber Luther noch einmal anders $\mathrm{zu}$ bewertende Bedeutung für die Einheit der Kirche herauszustellen, beginnt die katholische Theologie immer stärker zu begreifen, ohne dabei Luthers eigene Rolle demgegenüber abzuwerten. Im Lichte der gegenwärtigen Einigungsbemühungen zwischen Lutherischem Weltbund und römisch-katholischer Kirche erscheint Melanchthon unter den heutigen Voraussetzungen bedacht - wirklich als ein Ökumeniker seiner Zeit, der - unter den damaligen historischen Voraussetzungen - die allerletzten Möglichkeiten für die Bewahrung der Einheit der Kirche auszuloten suchte. Der Versuch einer Versöhnung auf dem Reichstag zu Augsburg misslang freilich, es kam im Laufe der späteren Jahrzehnte zur endgültigen Trennung; die Gegensätze in Lehre und Praxis der Kirchen verhärteten sich in der Folge zusehends. Der über Jahrhunderte erfolgte Prozess der Konfessionalisierung der Bekenntnisse hat zu einer solchen Verhärtung beigetragen. Schließlich zerbrach der in der Confessio Augustana noch enthaltene Einigkeitsversuch gerade an der Kirchenfrage. Obwohl das Trienter Konzil keine bedeutsamen Aussagen zur Ekklesiologie hervorbrachte, liegt die ,antireformatorische Spitze des Konzils jedoch in der Betonung des Evangelium in Ecclesia“63. Das wird vor allem in der engen Anbindung der Heiligen Schrift an die Tradition der Kirche erkennbar. Es bedurfte einer langen Zeit, um die Notwendigkeit einer erneuten Suche nach der Einheit einzusehen. Dabei musste man jedoch nicht gänzlich neu anfangen. Erst jetzt wird vollends klar, wie sehr sich der von Melanchthon zu seiner Zeit bereits markierte Weg zur Einheit der Kirche als fruchtbar erweist. Diese Einsicht ergibt

61 Einheit vor uns. Bericht der Gemeinsamen Römisch-katholischen/Evangelisch-lutherischen Kommission, Nr. 3 (DwÜ 2, 453).

62 Kirche und Rechtfertigung, Nr. 170 f. (DwÜ 3, 374f.).

63 W. Kasper, Das Kirchenverständnis der Confessio Augustana, 409. 
sich aber erst im Rückblick. Für Melanchthon zentral ist die Einsicht in den fortwährenden Konsens der einen katholischen Kirche. ${ }^{64}$ Im Blick auf das Wirken Melanchthons lässt sich dann formulieren: Die aus der Reformation hervorgegangenen Kirchen und Gemeinschaften haben die ursprüngliche Katholizität gesucht und suchen sie noch. ${ }^{65}$

Auch in einer weiteren Frage zeigt sich eine überraschende Kontinuität. Folgt man bereitwillig den Anforderungen des ökumenischen Dialogs, indem man die Verbundenheit von Rechtfertigung und Kirche, ohne die offenkundigen Differenzen $\mathrm{zu}$ leugnen, besonders hervorhebt, so lässt sich eine bemerkenswerte Kontinuität „nach hinten“ feststellen. Den Reformatoren war das Thema einer rechtfertigungstheologischen Konzentration der Ekklesiologie vor die Füße gelegt worden, wie die Forschungen zu Johannes Eck und die enzyklopädischen Hinweise der spätmittelalterlichen Theologie belegen. Insoweit bewegten sie sich in einem ihnen durch die spätmittelalterliche Theologie bereits vorbestimmten Rahmen. Die hier vorgelegte Analyse der CA lässt diesen rechtfertigungstheologischen Anspruch der Reformatoren deutlich hervortreten. Bewegten sich die Wittenberger Reformtheologen damit innerhalb einer, allerdings in einem größeren Rahmen zu beurteilenden „konfessorischen“ Katholizität oder sprengten sie diese? Die Differenz, die hier ausgemacht werden konnte, zeigt sich zwischen der mittelalterlichen Rezeption der patristischen Grundeinstellung, der für den Zusammenhang von Kirche und Eucharistie spricht, und der Neuausrichtung der Ekklesiologie im Zusammenhang mit der Heilsfrage. Diese Konzentration haben die Wittenberger Reformtheologen ohne Zweifel vorgenommen. ${ }^{66}$ Diesen Zusammenhang haben die Confutatio und die auf Trient folgende Theologie auch nicht bestritten. Erst das Zweite Vatikanische Konzil restituiert den eucharistisch-sakramentalen Kontext der Ekklesiologie in der Kirchenkonstitution Lumen Gentium.

Ganz in der Tradition dieser schon historisch nicht zu bestreitenden Übereinstimmung hält die Gemeinsame Erklärung zur Rechtfertigungslehre fest:

Unser Konsens in Grundwahrheiten der Rechtfertigungslehre muss sich im Leben und in der Lehre der Kirche auswirken und bewähren. Im Blick darauf gibt es noch Fragen von unterschiedlichem Gewicht, die weiterer Klärung bedürfen: [...] das Verhältnis von Wort Gottes

64 V. Pfnür, Die Einheit der Kirche in der Sicht Melanchthons, in: G. Frank/S. Meier-Oeser (Hg.), Konfrontation und Dialog. Philipp Melanchthons Beitrag zu einer ökumenischen Hermeneutik, Leipzig 2006, 123.

65 J. Ratzinger, Was eint und was trennt die Konfessionen?, in: ders., Gesammelte Schriften, Bd. 8/2, Freiburg i.B. 2010, 837.

66 Dazu J. Rahner, Creatura Evangelii. Zum Verhältnis von Rechtfertigung und Kirche, Freiburg i.B. 2005. 
und kirchlicher Lehre sowie die Lehre von der Kirche, von der Autorität in ihr, von ihrer Einheit, vom Amt und von den Sakramenten ${ }^{67}$.

Es handelt sich hier um offene Fragen, die nicht bereits mit dem erzielten Konsens in Grundfragen der Rechtfertigungslehre beantwortet sind. Insoweit muss die rechtfertigungstheologische Fragestellung dahingehend ekklesiologisch erweitert werden. Insbesondere muss gefragt werden, ob und wie das erreichte Verständnis in der Rechtfertigungslehre eine tragfähige Grundlage für eine Klärung der offen gebliebenen Fragen bildet.

Was könnte hier „tragfähige Grundlage“ heißen? Insoweit die Rechtfertigungslehre Maßstab oder Prüfstein des christlichen Glaubens ist, ${ }^{68}$ darf keine Lehre diesem Kriterium widersprechen. Die Rechtfertigungslehre führt ihre Bedeutung im Gesamtzusammenhang des grundlegenden trinitarischen Glaubensbekenntnisses der Kirche aus. Wenn die Rechtfertigungslehre Kriterium der ganzen Theologie ist, dann besteht also ein innerer Zusammenhang zwischen dieser Lehre und den anderen für das Kirchesein der Kirche notwendigen und grundlegenden Dimensionen. Der Konsens muss sich also bewähren in den folgenden Bereichen der Sakramente, des Amtes, der Kirche selbst. Also müssen wir die Frage stellen, was von der Rechtfertigungslehre in die Ekklesiologie hineinführt. Hier lautet nun die grundlegende These:

\begin{abstract}
Alles, was über das Wesen der Kirche, über die Heilsmittel und über das der Kirche eingestiftete Amt geglaubt und gelehrt wird, muß im Heilsgeschehen selbst begründet sein und vom Rechtfertigungsglauben als Empfang und Aneignung des Heilsgeschehens geprägt sein. Entsprechend muß auch alles, was über Wesen und Wirkung der Rechtfertigung geglaubt und gelehrt wird, im Kontext der Aussagen über die Kirche, die Heilsmittel und das der Kirche eingestiftete Amt verstanden werden. ${ }^{69}$
\end{abstract}

Das lutherisch-katholische Dokument Kirche und Rechtfertigung macht hierzu drei Grundüberzeugungen aus:

1. Die Kirche lebt vom Evangelium als „einem wesentlich externen Wort“ Gottes. Die Kirche geht aus dem Evangelium hervor, andererseits ergeht das Evangelium in der Kirche und ruft in die Gemeinschaft der Kirche. Diese externe Zusage ergeht in Wort und Sakrament. ${ }^{70}$

67 Gemeinsame Erklärung zur Rechtfertigungslehre des Lutherischen Weltbundes und der Katholischen Kirche, Nr. 43 (DwÜ 3, 430).

68 So heißt es im Annex der „Gemeinsamen Erklärung zur Rechtfertigungslehre“, Nr. 3 (DwÜ 3, 440).

69 Kirche und Rechtfertigung, Nr. 168 (DwÜ 3, 373f.).

70 Kirche und Rechtfertigung, Nr. 170 (DwÜ 3, 374). 
2. Das Evangelium ist wahrhaft schöpferisches Wort Gottes, die Kirche folglich creatura verbi divini. Insoweit ist die Kirche immer vom Evangelium unterschieden, aus dem sie hervorgeht und zu dem sie hinführt. Das Evangelium ist für alle Zeiten der Ursprung jedweden Lebens für die Kirche. ${ }^{71}$

3. Gott ist seinem Worte und seiner Verheißung immer treu. Insofern ist die Kirche in ihrer geschichtlichen Gestalt durch Strukturen Ausdruck der Kontinuität Gottes mit ihr. Katholisch gelesen heißt das: Christus hat in seiner Kirche verschiedene Dienstämter eingesetzt (ministeria), die Gottes Sendung bezeugen. ${ }^{72}$

Zieht man nun hierzu das deutsche lutherisch-katholische Dialogdokument Communio Sanctorum ${ }^{73}$ heran, wird folgendes erkennbar: Was in Kirche und Rechtfertigung in der lutherischen Terminologie zum Ausdruck gebracht wird, erfolgt in Communio Sanctorum ausdrücklich in eher katholischer Begrifflichkeit. Hier werden die jeweiligen Schwerpunkte in Begriffen der Gemeinschaft (communio sanctorum) zum Ausdruck gebracht. So heißt es explizit: Gemeinschaft der Heiligen durch Wort und Sakrament, Gemeinschaft der zum Dienst Berufenen, und schließlich begründend: Gemeinschaft der aus Gnade Geheiligten. Gemeinsam gelesen ergibt sich die Erkenntnis: in dem einen Dokument wird die gemeinsame Grundüberzeugung von der engen Verhältnisbestimmung von Rechtfertigung und Kirche durch rechtfertigungstheologische Termini, in dem anderen durch Gemeinschaftsaspekte betont. Die konfessionsspezifischen Leitperspektiven werden so gelesen und dargestellt, dass sie die jeweils andere Perspektive einbeziehen und nicht ausschließen. Werden so die unterschiedlichen konfessionsspezifischen Perspektiven wirklich miteinander verknüpft oder nur lose (,semantisch“) miteinander verbunden?

Der neuerdings vorliegende Bericht über Kirche und Kirchengemeinschaft ${ }^{74}$ führt hierzu nun die These aus, dass eine wesentliche Übereinstimmung in der Überzeugung besteht, dass Rechtfertigung und Kirche, Gnadengeschehen wie die Gemeinschaft der Glaubenden im Heilswerk Christi selbst begründet und daher gleichursprünglich seien. Das erlösende Handeln Gottes zielt auf den

71 Kirche und Rechtfertigung, Nr. 171 (DwÜ 3, 375).

72 Kirche und Rechtfertigung, Nr. 172 (DwÜ 3, 375).

73 Bilaterale Arbeitsgruppe der Deutschen Bischofskonferenz und der Kirchenleitung der Vereinigten Evangelisch-Lutherischen Kirche Deutschlands, Communio Sanctorum. Die Kirche als Gemeinschaft der Heiligen, Paderborn/Frankfurt a. M. 2000.

74 Ch. Schad/K.-H. Wiesemann (Hg.), Bericht über Kirche und Kirchengemeinschaft. Ergebnis einer Konsultationsreihe im Auftrag der Gemeinschaft Evangelischer Kirchen in Europa und des Päpstlichen Rats zur Förderung der Einheit der Christen, Paderborn/Leipzig 2019. 
antwortenden Glauben der Menschen wie es diese untereinander zusammenführt in der Gemeinschaft des Heils. Kirche und Rechtfertigung gehören unlösbar zusammen (Nr. 42). „Mit der Rede von der Gleichursprünglichkeit wird vielmehr betont, dass die Kirche immer schon als die Gemeinschaft der Gerechtfertigten communio sanctorum ist und es keine Rechtfertigung gibt ohne Eingliederung in die Kirche, welche in der Taufe in den Leib Christi vollzogen und dargestellt wird“ (Nr. 45). Es ist nicht zu übersehen, dass innerhalb des lutherisch-katholischen Dialogs die Gewichte zwischen der auf das glaubende Individuum bezogenen Heilsfrage, welche die Rechtfertigungslehre stellt, und der Frage nach dem heilsinstrumentalen Charakter der Kirche, die in der Ekklesiologie verhandelt wird, neu austariert werden sollen. Je stärker die katholische Theologie die sakramentale Dimension der Kirche betont, umso mehr beharrt die lutherische Theologie auf der kriteriologischen Bedeutung der Rechtfertigungslehre für die gesamte Theologie. So ist die Feststellung zu verstehen: „Die Rede von der Gleichursprünglichkeit von Rechtfertigung und Kirche impliziert die notwendige Unterscheidung zwischen Kirche und Christus, Kirche und Heilsgeschehen. Wenn von der Sakramentalität der Kirche [...] gesprochen wird, dient diese Rede genau dieser Unterscheidung und der daraus resultierenden Zuordnung. “75 Deswegen betont katholische Theologie, dass die Kirche, wie es in Lumen Gentium heißt, veluti sacramentum (LG 1) ist, die Kirche eben nicht Sakrament ist wie die anderen Sakramente. „Sie ist und wirkt sakramental, d.h. sie hat an Struktur und Wesen der Sakramente teil."76 Die Unterscheidung zwischen Christus und der Kirche ist das entscheidende Leitmotiv der Rede von der Sakramentalität der Kirche. Ist diese Einschätzung zu Recht erfolgt? Wenn sie zu Recht erfolgt ist, dann wäre hiermit erwiesen, dass der in der CA enthaltene Anspruch auf die rechtfertigungstheologische Bestimmung der Kirche von katholischer und lutherischer Überzeugung im Konsens getragen wird.

Wenn für die Kirche und ihre Sendung konstitutiv sind: das ihr überlieferte Wort Gottes, die Sakramente des Glaubens und das Amt der Verkündigung, dann bilden diese drei Elemente und Güter der Heiligung und Wahrheit die Grundlage für das Wesen und die Sendung der Kirche. Insofern impliziert der Grundkonsens in der Rechtfertigungslehre die notwendige Aufgabe, einen Konsens in den Fragen der Sakramente und im kirchlichen Amt festzustellen, der diesem Grundkonsens in der Rechtfertigungslehre entspricht. Ist mit dem Grundkonsens in der Rechtfertigungslehre schon der Konsens in Fragen der Sakramente und des Amtes

75 Bericht über Kirche und Kirchengemeinschaft, Nr. 51, 40. 76 Bericht über Kirche und Kirchengemeinschaft, Nr. 51, 40. 
mitgegeben oder muss er erst hergestellt werden? Freilich stellt sich mit der Rolle der Sakramentalität der Kirche eine entscheidende weiter reichende Frage: Welche Ordnung und Struktur entspricht diesem Grundprinzip der Sakramentalität? Eine Antwort hierzu hält schon das deutsche lutherisch-katholische Dokument „Kirchengemeinschaft in Wort und Sakrament“ bereit, wenn es heißt:

Beide Kirchen stimmen darin überein, dass für die Einheit der Kirche Wort und Sakrament konstitutiv sind und dass für die Wortverkündigung und für die Sakramentenverwaltung das Amt gestiftet ist und Ämter in der Kirche notwendig sind (CA V, VII; XIV; LG 14). Kontrovers ist zwischen uns aber, ob und inwiefern zu diesem Amt der Leitung von Wortverkündigung und Sakramentsverwaltung eine bestimmte geschichtliche Gestalt gehört, um gültiges Zeichen der Einheit sein zu können - eine Gestalt, die deshalb dann verbindlich ist ${ }^{77}$.

Jahrzehnte später kann vor der Folie der Kirche als Communio Sanctorum zwar ein weiter führender Konsens in Fragen der Bezeugungsinstanzen und des Petrusamtes festgestellt werden, aber eine zureichende Antwort auf die Frage nach in der Rechtfertigungsfrage mitenthaltenen „Sakramentalität der Kirche“ bleibt aus. Noch einmal zwei Jahrzehnte später formuliert der Bericht der Lutherisch-Katholischen Dialog-Kommission für Finnland einen „wachsenden lutherisch-katholischen Konsens über Kirche, Eucharistie und Amt im Kontext der gemeinsamen sakramentalen Ekklesiologie“78. Dieser Konsens schließt ausdrücklich „die konstitutive Bedeutung von Amt, Wort und Sakrament und die Kontinuität der Kirche ,für immer“"79 ein. Hier wird die einheitsstiftende Überzeugung der CA zur Geltung gebracht. Ob sie tragfähig erscheint, muss der zukünftige Dialog erweisen.

77 Bilaterale Arbeitsgruppe der Deutschen Bischofskonferenz und der Kirchenleitung der Vereinigten Evangelisch-Lutherischen Kirche Deutschlands, Kirchengemeinschaft in Wort und Sakrament, Paderborn/Hannover 1984, 15.

78 Wachsende Gemeinschaft. Erklärung über Kirche, Eucharistie und Amt. Bericht der Lutherisch-Katholischen Dialog-Kommission für Finnland. Evangelisch-Lutherische Kirche Finnlands/ Katholische Kirche in Finnland, Helsinki 2017, Paderborn/Leipzig 2018, Nr. 363, 208.

79 Wachsende Gemeinschaft, Nr. 35, 40. 
